\title{
Modeling Manifest Huntington's Disease Prevalence Using Diagnosed Incidence and Survival Time
}

\author{
Valerie Crowell ${ }^{a}$ Richard Houghton ${ }^{b}$ Akanksha Tomar ${ }^{c}$ Tricia Fernandes ${ }^{d}$ \\ Ferdinando Squitieri $\mathrm{i}^{\mathrm{a}}$

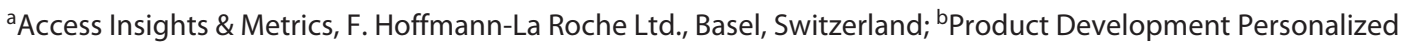 \\ Healthcare, F. Hoffmann-La Roche Ltd., Basel, Switzerland; ' ZS Associates International Inc., Frankfurt, Germany; ${ }^{\text {dZS }}$

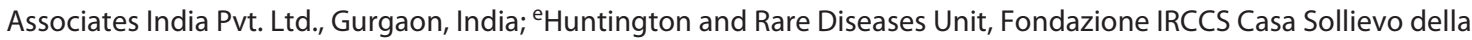 \\ Sofferenza Research Hospital, San Giovanni Rotondo, Italy
}

\section{Keywords}

Epidemiology · Huntington's disease $\cdot$ Neurodegeneration · Disease progression $\cdot$ Shoulson-Fahn stage

\begin{abstract}
Introduction: Understanding the epidemiology of Huntington's disease (HD) is key to assessing disease burden and the healthcare resources required to meet patients' needs. We aimed to develop and validate a model to estimate the diagnosed prevalence of manifest HD by the Shoulson-Fahn stage. Methods: A literature review identified epidemiological data from Brazil, Canada, France, Germany, Italy, Spain, the UK, and the USA. Data on staging distribution at diagnosis, progression, and mortality were derived from Enroll-HD. Newly diagnosed patients with manifest HD were simulated by applying annual diagnosed incidence rates to the total population in each country, each year from 1950 onwards. The number of diagnosed prevalent patients from the previous year who remained in each stage was estimated in line with the probability of death or progression. Diagnosed prevalence in 2020 was estimated as the sum of simulated patients, from all the incident cohorts, still alive. Results: The model estimates that in 2020 , there were 66,787 individuals
\end{abstract}

(C) 2021 The Author(s)

Published by S. Karger AG, Basel

This is an Open Access article licensed under the Creative Commons Attribution-NonCommercial-4.0 International License (CC BY-NC) (http://www.karger.com/Services/OpenAccessLicense), applicable to the online version of the article only. Usage and distribution for commercial purposes requires written permission. diagnosed with HD in the 8 included countries, of whom 62 $63 \%$ were in Shoulson-Fahn stages 1 and 2 (with less severely limited functional capacity than those in stages 3-5). Diagnosed prevalence is estimated to be 8.2-9.0 per 100,000 in the USA, Canada, and the 5 included European countries and 3.5 per 100,000 in Brazil. Conclusion: The modeled estimates generally accord with the previously published data. This analysis contributes to better understanding of the epidemiology of $\mathrm{HD}$ and highlights areas of uncertainty.

(C) 2021 The Author(s)

Published by S. Karger AG, Basel

\section{Introduction}

Huntington's disease (HD) is a rare, progressive, genetic, neurodegenerative disease that is ultimately fatal and has devastating effects on the lives of patients and their families $[1,2]$. Despite identification of the genetic mutation underlying HD over 20 years ago, current treatment options focus only on symptomatic management [3].

Several scales have been developed to aid diagnosis and to follow patients systematically after diagnosis of 


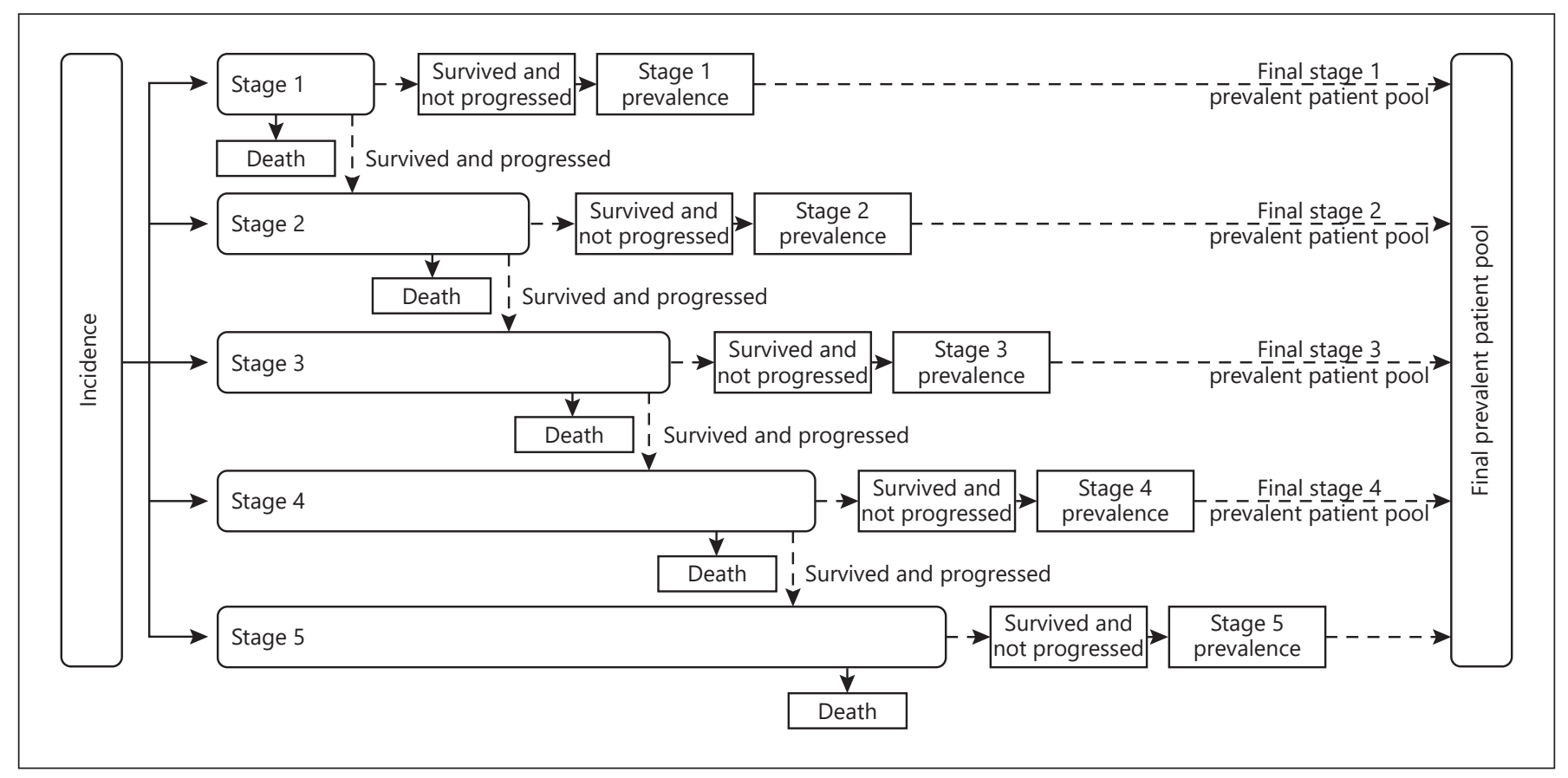

Fig. 1. Flow of patients between Shoulson-Fahn TFC-based stages to obtain the final pool of prevalent patients. TFC, Total Functional Capacity.

HD. Two of the most used are the Unified Huntington Disease Rating Scale $[4,5]$ and 1 of its component scales, the Total Functional Capacity (TFC) rating scale [6]. The Shoulson-Fahn system ranks independence in daily activities on a scale from stage 1 to stage 5 , where stage 5 represents the most severely limited functional capacity $[6,7]$. These stages are based on TFC scores (stage 1: 1113 , stage $2: 7-10$, stage $3: 3-6$, stage $4: 1-2$, and stage $5: 0$ ).

Understanding the manifest HD population is key to evaluate disease burden and the resources required to meet patients' needs. There are limited published data on the incidence and prevalence of HD (unless otherwise stated, incidence and prevalence refer to diagnosed incidence and prevalence throughout this report). Data on the incidence of HD suggest that it is in the range of $0.1-$ 0.7 per 100,000 people [2, 8-19]. Higher estimates are commonly derived from insurance claims data compared with other sources $[20,21]$. Available data suggest that the prevalence of HD is approximately 10 cases per 100,000 people, with higher prevalence in North America, Northwestern Europe, the Middle East, and Australia, where estimates range from 5.96 to 13.70 cases per 100,000 people $[19,22-25]$ than in Asia, where the prevalence is estimated at $0.41-0.70$ cases per 100,000 people [26]. However, the real prevalence, including undiagnosed individ- uals, may be higher than these estimates for a variety of reasons [27].

The causal structure of disease dictates that incidence, prevalence, and mortality are interrelated [28]. Incidence must precede prevalence, and cause-specific mortality may only follow disease. Based on this premise, an incidence-prevalence-mortality model can be developed to supplement and/or evaluate agreement between available data and to explore shifting epidemiological dynamics [28-30]. The objective of the study described in this report was to develop an epidemiological model to estimate the prevalence of manifest HD by the Shoulson-Fahn stage and validate this with population-based prevalence estimates.

\section{Methods}

Study Design Overview

Data on the incidence of HD were derived from a review of published HD epidemiology data (described in the online suppl. material; see www.karger.com/doi/10.1159/000516767 for all online suppl. material). Data on the staging distribution at incidence of $\mathrm{HD}$ and on the survival and progression of patients by the Shoulson-Fahn stage were derived from an observational study of patients with HD (Enroll-HD: ClinicalTrials.gov identifier: NCT01574053). The study design is shown in online suppl. Fig. 1. 
Table 1. Estimated 2020 prevalence of HD in 8 countries, overall and by Shoulson-Fahn TFC-based stage, for high-, medium-, and lowincidence inputs

\begin{tabular}{|c|c|c|c|c|c|c|c|}
\hline $\begin{array}{l}\text { Country (population, } \\
\text { thousands) [31] }\end{array}$ & Case & $\begin{array}{l}\text { Estimated total diagnosed } \\
\text { prevalent patients with manifest } \\
\text { HD (prevalence per } 100,000)\end{array}$ & \multicolumn{5}{|c|}{$\begin{array}{l}\text { Estimated diagnosed prevalent patients with } \\
\text { manifest HD by Shoulson-Fahn stage (\% of total } \\
\text { diagnosed prevalent patients with manifest HD) }\end{array}$} \\
\hline \multirow[t]{2}{*}{ Brazil $(212,559)$} & High & $18,269(8.6)$ & 5,084 & 6,406 & 4,459 & 1,966 & 353 \\
\hline & Medium & $7,406(3.5)$ & 2,061 & 2,597 & 1,808 & 797 & 143 \\
\hline \multirow[t]{3}{*}{ Canada $(37,742)$} & High & $4,010(10.6)$ & 1,124 & 1,408 & 974 & 427 & 76 \\
\hline & Medium & $3,077(8.2)$ & 862 & 1,080 & 747 & 328 & 59 \\
\hline & Low & $2,721(7.2)$ & 762 & 955 & 661 & 291 & 53 \\
\hline \multirow[t]{3}{*}{ France $(65,274)$} & High & $7,232(11.1)$ & 1,976 & 2,529 & 1,785 & 797 & 145 \\
\hline & Medium & $5,789(8.9)$ & 1,580 & 2,023 & 1,429 & 640 & 117 \\
\hline & Low & $4,924(7.5)$ & 1,343 & 1,720 & 1,216 & 545 & 100 \\
\hline \multirow[t]{3}{*}{ Italy $(60,462)$} & High & $6,799(11.2)$ & 1,843 & 2,375 & 1,685 & 757 & 139 \\
\hline & Medium & $5,443(9.0)$ & 1,475 & 1,900 & 1,349 & 607 & 112 \\
\hline & Low & $2,272(3.8)$ & 614 & 792 & 563 & 255 & 47 \\
\hline \multirow[t]{3}{*}{ Spain $(46,755)$} & High & $5,256(11.2)$ & 1,423 & 1,842 & 1,307 & 580 & 104 \\
\hline & Medium & $4,207(9.0)$ & 1,138 & 1,474 & 1,046 & 465 & 84 \\
\hline & Low & 2,751 (5.9) & 743 & 962 & 684 & 306 & 56 \\
\hline \multirow[t]{3}{*}{ UK $(67,886)$} & High & $7,364(10.9)$ & 2,038 & 2,581 & 1,803 & 798 & 145 \\
\hline & Medium & $5,895(8.7)$ & 1,630 & 2,065 & 1,443 & 640 & 117 \\
\hline & Low & $5,014(7.4)$ & 1,386 & 1,755 & 1,228 & 546 & 100 \\
\hline
\end{tabular}

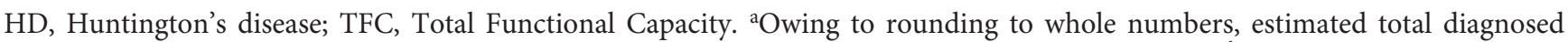
prevalent patients with manifest HD may differ slightly from the sum for Shoulson-Fahn stages $1-5$. ${ }^{\text {b}}$ Modeled prevalent staging distributions vary slightly across countries because of differences in population demographics.

\section{Epidemiological Model of HD}

An epidemiological model of manifest HD to estimate prevalence, dependent on probabilities of progression and survival by stage, was developed using Microsoft Excel.

The model included several of the most populous countries in the world in which a high proportion of the population has Western European ancestry. Newly diagnosed patients with manifest HD were simulated by applying incidence rates to the total population in each country, each year [31]. Incidence was extrapolated when countryspecific data were unavailable (shown in online suppl. Table 1) [1017]. In each region, a base incidence rate was applied in 1950. The base rate was increased linearly until the peak rate, which was designated as being in 2006, the average year of the medium-case estimates of peak incidence. This linear increase was effected in order to avoid overestimating current prevalence, based on the assumption that incidence increased over the last generation due to increased awareness and improved testing relative to previous decades [32].

Prevalence of Huntington's Disease: A Model
The incident patients in each year were allocated across Shoulson-Fahn TFC-based stages [6] according to information on the stage at diagnosis from patients with premanifest HD in EnrollHD whose disease became manifest during the observation period. In the simulation model, each year, some patients remained in the same stage, some progressed to the next stage, some left the cohort owing to death, and some new incident patients joined the cohort (shown in Fig. 1). In each simulated year, the number of prevalent patients from the previous year who remained in each stage was estimated in line with the time-varying probability of death or progression out of that stage. The probability of progression was applied in each annual cohort to estimate the number of patients who progressed to the next stage. To generate a stable prevalent pool of patients, the simulation was undertaken for the period between 1950 and 2020. The total prevalent population in each stage at a given time point was calculated from the sum of the numbers of patients currently in that stage from each annual incident cohort. 
Fig. 2. Estimated prevalence (per 100,000) of manifest HD, 2000-2020 in the USA (a) and in France, Germany, Italy, Spain, and the UK combined (b), and comparison with previously published estimates [11, $15,16,20,23-25,40,41]$. HD, Huntington's disease.

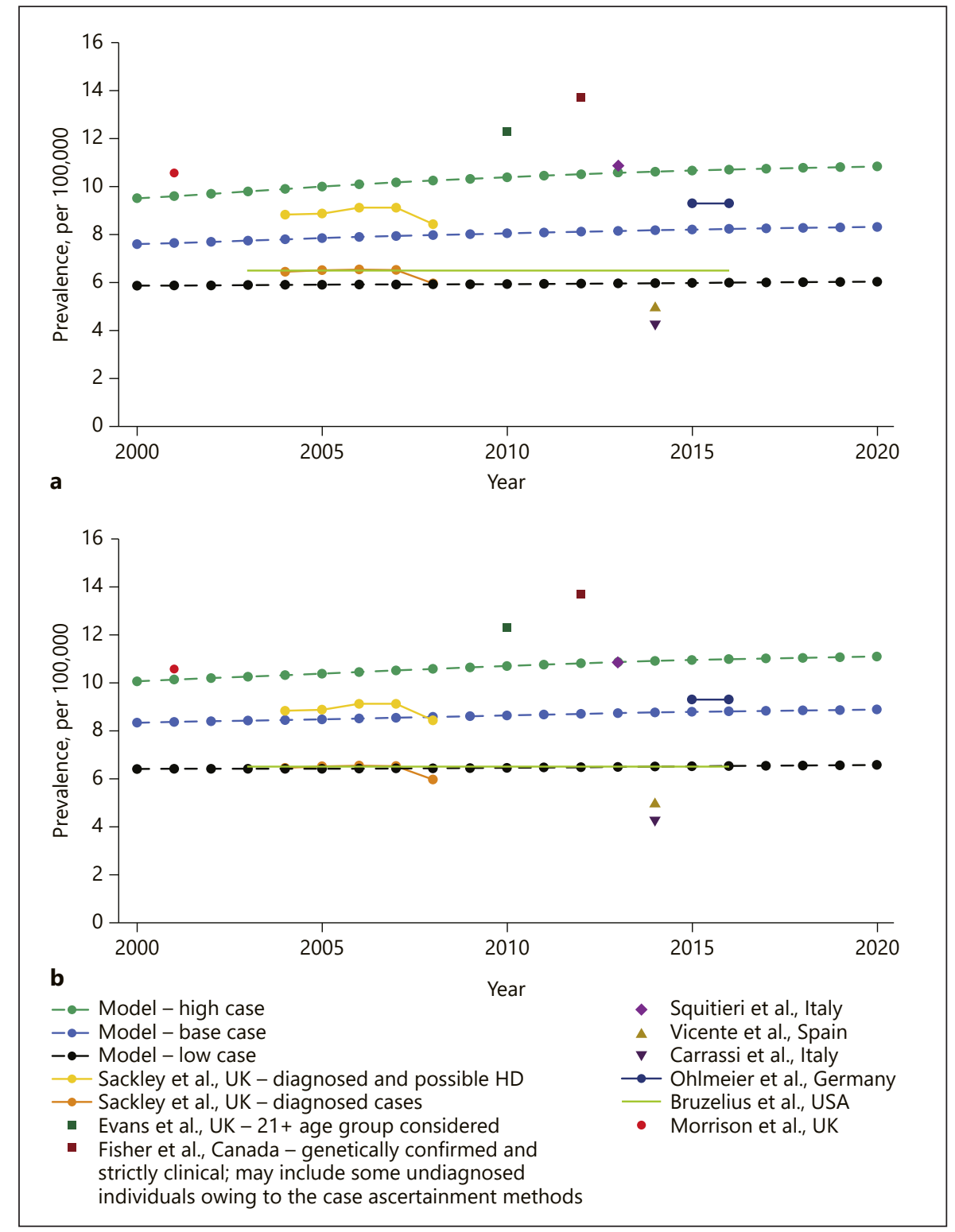

Staging Distribution at Incidence and Survival (Analysis of Enroll-HD)

Enroll-HD is a global clinical research platform designed to facilitate clinical research in HD [33]. Core datasets are collected annually from all research participants as part of this multicenter longitudinal observational study. Data are monitored for quality and accuracy using a risk-based monitoring approach. All sites are required to obtain and maintain local ethical approval. The study population includes patients and family members who carry or are at risk for the HD gene expansion mutation and control participants who do not carry the mutation. The gene-positive population is divided into individuals with manifest HD and those with premanifest HD.

Data from Enroll-HD Periodic Dataset 4 version 2.0 (data cutoff October 31, 2018) were used in developing the staging distribu- tion of incident patients and progression through stages in the model. The full Periodic Dataset comprised the clinical data from 15,301 participants.

The staging distribution of incident patients (at diagnosis) was restricted to patients whose disease was premanifest at baseline and became manifest during follow-up to ensure that the stage distribution at diagnosis of manifest HD was captured. Kaplan-Meier survival curves for overall survival (OS; death) and progressionfree survival (PFS) were estimated by the Shoulson-Fahn stage. Progression was defined as moving out of the current stage (to a more advanced stage or death). To avoid left censoring in the data, estimates were based on total time spent in a stage before moving to the next stage, rather than time from first enrollment in the database. As such, the analysis was restricted to patients whose disease either became manifest during follow-up or changed from 1 
Table 2. Characteristics of the Enroll-HD cohort by Shoulson-Fahn TFC-based stage

\begin{tabular}{|c|c|c|c|c|c|}
\hline & \multicolumn{5}{|c|}{ Shoulson-Fahn stage } \\
\hline & 1 & 2 & 3 & 4 & 5 \\
\hline Sex, male, $n(\%)$ & $123(46.2)$ & $457(51.2)$ & $467(49.5)$ & $209(46.3)$ & $58(34.1)$ \\
\hline \multicolumn{6}{|l|}{ Current age, $n(\%)$} \\
\hline$<45$ years & $120(45.1)$ & $241(27.0)$ & $216(22.9)$ & $75(16.6)$ & $26(15.3)$ \\
\hline$>61$ years & $24(9.0)$ & $188(21.1)$ & $288(30.5)$ & $165(36.6)$ & $63(37.1)$ \\
\hline Mean current age, years (SD) & $46.52(12.16)$ & $51.75(12.29)$ & $54.43(12.90)$ & $56.53(12.55)$ & $56.90(13.01)$ \\
\hline Diagnosed at $<18$ years old, $n(\%)^{\mathrm{a}}$ & $2(0.8)$ & $12(1.3)$ & $14(1.5)$ & $11(2.4)$ & $2(1.2)$ \\
\hline Mean CAG tract length, repeats (SD) & $43.44(3.18)$ & $43.92(3.72)$ & $44.32(4.31)$ & $44.84(4.90)$ & $45.18(4.72)$ \\
\hline Mean CAG-age product (SD) & $426.35(81.43)$ & $496.03(84.73)$ & $536.51(94.03)$ & $582.76(105.44)$ & $605.45(99.90)$ \\
\hline \multicolumn{6}{|l|}{ Region, $n(\%)$} \\
\hline \multicolumn{6}{|l|}{ Race, $n(\%)$} \\
\hline Asian & $2(0.8)$ & $6(0.7)$ & $7(0.7)$ & $2(0.4)$ & 0 \\
\hline Black & $1(0.4)$ & $5(0.6)$ & $11(1.2)$ & $6(1.3)$ & $3(1.8)$ \\
\hline Hispanic or Latino & $3(1.1)$ & $14(1.6)$ & $23(2.4)$ & $8(1.8)$ & $4(2.4)$ \\
\hline Mixed & $6(2.3)$ & $7(0.8)$ & $8(0.8)$ & $3(0.7)$ & 0 \\
\hline Native American & 0 & $2(0.2)$ & $4(0.4)$ & $3(0.7)$ & 0 \\
\hline Other & $4(1.5)$ & $14^{\mathrm{b}}(1.6)$ & $11(1.2)$ & $7(1.6)$ & $1(0.6)$ \\
\hline White & $250(94.0)$ & $844(94.6)$ & $879(93.2)$ & $422(93.6)$ & $162(95.3)$ \\
\hline
\end{tabular}

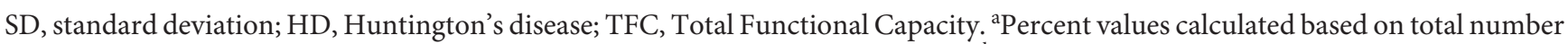
of patients; age at diagnosis data were unavailable for a small proportion of patients. ' Includes 1 individual for whom information on race was unavailable.

manifest baseline stage to another during follow-up. In all time-toevent analyses, if no progression or death occurred, patient time was censored at the last visit at which TFC was measured. A Cox proportional hazards model was used to test for differences in PFS by stage, with stage 1 as the reference group. Parametric survival analysis methods were used to derive complete time-to-event curves, and a one-way sensitivity analysis varying incidence inputs was conducted, as described in the online suppl. Material. The survival models by stage were not adjusted for any covariates because estimates were intended to be representative of patients in the Enroll-HD database.

\section{Results}

\section{Model Outputs and Sensitivity Analyses}

Estimates of manifest HD prevalence in 2020 are shown in Table 1. Medium incidence-input prevalence rates are estimated to be $8.2-9.0$ per 100,000 in the USA, Canada, and the 5 included European countries and 3.5 per 100,000 in Brazil. The ranges of prevalence estimates for the USA, and for France, Germany, Italy, Spain, and the UK combined, are shown in Figure 2. The model estimates an overall prevalence of 66,787 individuals in the 8 included countries, of whom $62-63 \%$ are in ShoulsonFahn TFC-based stages 1 and 2. The modeled prevalence across all included countries in the sensitivity analyses was 48,028-94,102 individuals.

\section{Literature Review and Published HD Epidemiology Data}

Eight original studies were identified for inclusion [10-17]. An overview of these studies is provided in online suppl. Table 2. Additionally, an unpublished estimate of HD prevalence in Brazil was obtained from the Brazilian HD Patients' Association.

Based on the data identified, the base (1950) and peak (2006) incidences of manifest HD were estimated (shown in online suppl. Table 1). A medium base incidence of 0.50 per 100,000 people was applied across all included countries except Brazil where, owing to the 
Fig. 3. Parametric OS curves by ShoulsonFahn TFC-based stage and the PFS curve applied across all Shoulson-Fahn stages. TFC, Total Functional Capacity; PFS, progression-free survival; OS, overall survival.

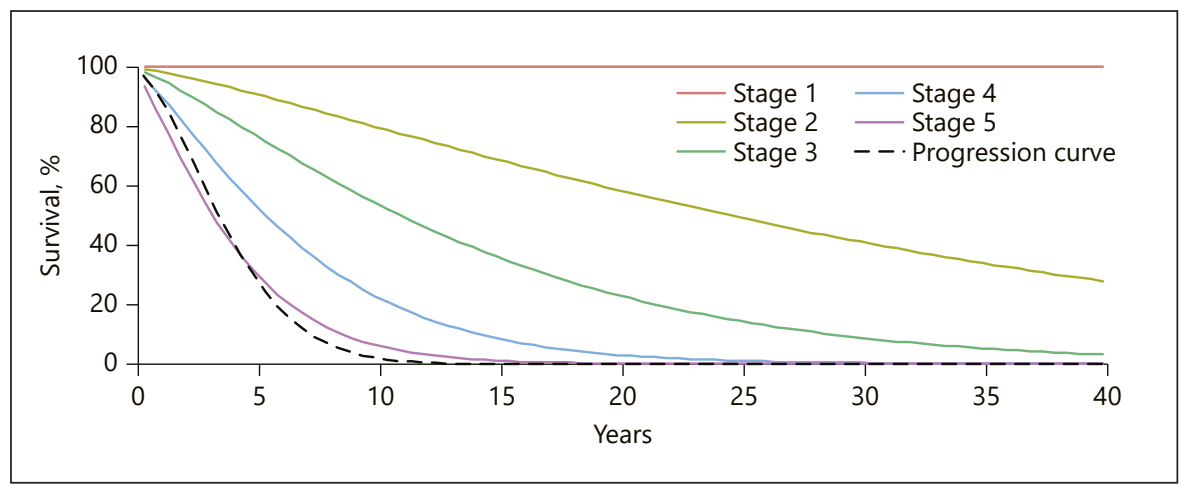

lack of incidence data, low- and medium-case incidences were back-calculated from prevalence. Across all scenarios, peak diagnosed incidence inputs ranged from a low value of 0.16 per 100,000 people [12] in Brazil to a high value of 0.90 per 100,000 in the other countries [15].

\section{Enroll-HD Cohort}

Demographic characteristics of the patients in the Enroll-HD cohort by Shoulson-Fahn stage are shown in Table 2. Most patients in the Enroll-HD cohort were diagnosed with manifest $\mathrm{HD}$ when in stage 1 . The proportions of incident cases of manifest HD in Enroll-HD were 75\% in stage $1,22 \%$ in stage 2 , and $3 \%$ in stage 3 .

A Cox proportional hazards model was used to analyze PFS by stage for the patients in Enroll-HD. The Cox model showed no marked, ordinal, or statistical difference for PFS between most stages and PFS was therefore assumed to be the same for all stages (see online suppl. Table 3; online suppl. Fig. 2).

The OS curves and PFS curve used in the model are shown in Figure 3. The best-fitting models were the loglogistic and the Gompertz for PFS and for OS, respectively (see online suppl. Table 4). The Weibull models were the second best-fitting models for both PFS and OS. The Weibull models were chosen as the final extrapolations based on the more plausible shape of the Weibull curves and their use together, despite slightly less good fit. The estimated median OS time for stage 1 was higher than clinically plausible, owing to the small number of patients who died in stage 1 in Enroll-HD. However, using the Weibull model, the estimated probability of progression reached $99 \%$ by 11.1 years, and the modeled estimate of OS from stage 2 was plausible. The estimated median PFS (time in stage) for all stages using the Weibull curve was 3.61 years.

\section{Discussion}

To our knowledge, this is the first incidence-prevalence-mortality model of HD using natural history data that takes into account differences in patients' functional capacity, scored using the Shoulson-Fahn TFC scale. The wide range of modeled prevalence in the sensitivity analyses $(48,028-94,102)$ highlights the uncertainty around HD epidemiological estimates. However, the estimates of prevalence across the selected countries, based on the model, are generally in accordance with those from the published literature $[8,11,15,24,25]$, indicating validity of the model. Furthermore, the estimated distributions of prevalence by Shoulson-Fahn stage are comparable to those included in previous reports [34-36].

Two meta-analyses estimated average HD prevalence in predominantly Caucasian populations of 5.70 (95\% CI: 4.42-7.35) per 100,000 and 9.71 (95\% CI: 9.32-10.12) per 100,000 using studies from 1985 to 2010 and since 1995, respectively $[8,37]$; a third systematic review concluded that study methods were too heterogeneous to pool their estimates [26]. Several recent epidemiological studies from the USA illustrate the variability in study populations and methods and ensuing challenges when interpreting results $[20,21,38]$. Nevertheless, HD prevalence is increasing, while incidence has been stable, over the past several decades at least $[13,26,37]$. This may be attributed to decreased stigma surrounding a diagnosis of $\mathrm{HD}$, leading to increased willingness of clinicians to include an HD diagnosis in the records of previously diagnosed patients [39] and/or overall increases in life expectancy that will likely be reflected in increased longevity for individuals with HD. A greater availability of effective symptomatic treatments for HD may also reduce mortality, thereby increasing prevalence. Incidence, stage at diagnosis and progression and survival in our model generally reflect recent values for these parameters, and our 
estimated prevalence rates are more in line with later empirical estimates. Our model allows exploration of these dynamics. However, uncertainty around existing epidemiological data informing these parameters means there is a need for more primary evidence in this area, including from more representative longitudinal studies, to allow firm conclusions to be drawn about the magnitude and drivers of HD epidemiological shifts. Owing to lack of data, the geographical coverage of our model was limited, and the modeled estimates for individual countries should be interpreted with caution.

Among the strengths of our study are the use of natural history data from a large cohort of patients with HD to support the modeling of the progression, OS, and PFS of the overall population with HD. Although several different factors may influence an individual's time to progression, average rates can be used to generate population-level prevalence estimates. Therefore, no stratification or adjustment by CAG tract length, age, or other variables that may influence progression or mortality was implemented. Moreover, the model was configured so that death did not compete with progression to the next stage, with progression being a composite of progression to the next stage or death.

A limitation of the model is that estimated stage at incidence, time in stage, and time to death are assumed as constant, based on data gathered in recent years and from a single source (Enroll-HD) analyzing a predominantly Caucasian European cohort. It is possible that the patients participating in Enroll-HD may not be representative of the general population with HD; for example, they may be diagnosed earlier in the course of the disease or be followed more closely, resulting in systematically different rates of progression or survival compared with the general population of individuals with HD. Furthermore, the year-long intervals between assessments might limit the granularity of the collected data. However, it was evident that very few patients skipped stages (online suppl. Table 5), and dates of death were accurately recorded. Owing to the short median follow-up in Enroll-HD, it was necessary to extrapolate the data to a longer duration, with resulting uncertainty.

\section{Conclusions}

Robust estimates of the prevalence of $\mathrm{HD}$ are required to allow better understanding of its impacts and to ensure adequate medical and societal resources are available. This analysis contributes to the better characterization of the epidemiology of manifest HD, highlights areas of uncertainty and evidence gaps, and enables dynamic predictions of prevalence based on incidence, progression, and mortality.

\section{Acknowledgements}

The authors thank those involved with Enroll-HD. Enroll-HD is a clinical research platform and longitudinal observational study for HD families intended to accelerate progress towards therapeutics; it is sponsored by CHDI Foundation, a nonprofit biomedical research organization exclusively dedicated to collaboratively developing therapeutics for HD. Enroll-HD would not be possible without the vital contribution of the research participants and their families. The authors thank the individuals who contributed to the collection of the Enroll-HD data (https://www.enroll-hd. org/acknowledgments/). The authors thank Isaac Gravestock of F. Hoffmann-La Roche Ltd, for quality checking Enroll-HD analysis results. The authors thank Colin Glen of Oxford PharmaGenesis, Oxford, UK, for providing medical writing support, which was funded by F. Hoffmann-La Roche Ltd, in accordance with Good Publication Practice (GPP3) guidelines (http://www.ismpp.org/ gpp3).

\section{Statement of Ethics}

Ethical approval was not required for this study.

\section{Conflict of Interest Statement}

V.C. and R.H. are employees of F. Hoffmann-La Roche Ltd. A.T. is an employee of ZS Associates International Inc. T.F. is an employee of ZS Associates India Pvt. Ltd. F.S. has provided consulting services and advisory board functions to F. Hoffmann-La Roche Ltd, Novartis, Pfizer, PTC Therapeutics, Teva, UCB, and the Oman Ministry of Health - Sultanate of Oman. He is cofounder, scientific director, and consultant of the not-for-profit organization Italian League for Research on HD (LIRH Foundation).

\section{Funding Sources}

This study was funded by F. Hoffman-La Roche Ltd.

\section{Author Contributions}

V.C. and R.H. conceived and designed the study and conducted the research, including statistical analyses, in collaboration with A.T. and T.F., with review and critique by F.S. V.C. wrote the first draft of this manuscript, with subsequent review and critique from R.H., A.T., T.F., and F.S. 


\section{References}

1 Roos RA. Huntington's disease: a clinical review. Orphanet J Rare Dis. 2010 Dec 20;5:40.

2 Bates GP, Dorsey R, Gusella JF, Hayden MR, Kay C, Leavitt BR, et al. Huntington disease. Nat Rev Dis Primers. 2015 Apr 23;1:15005.

3 Coppen EM, Roos RA. Current pharmacological approaches to reduce chorea in Huntington's disease. Drugs. 2017 Jan;77(1):29-46.

4 Huntington Study Group. [Accessed January 8, 2021] Available from:https://huntingtonstudygroup.org/.

5 Huntington Study Group. Unified Huntington's disease rating scale: reliability and consistency. Mov Disord. 1996 Mar;11(2):136-42.

6 Shoulson I, Fahn S. Huntington disease: clinical care and evaluation. Neurology. 1979 Jan; 29(1):1-3.

7 Paulsen JS, Wang C, Duff K, Barker R, Nance $\mathrm{M}$, Beglinger L, et al. Challenges assessing clinical endpoints in early Huntington disease. Mov Disord. 2010 Nov 15;25(15):2595603.

8 Pringsheim T, Wiltshire K, Day L, Dykeman J, Steeves T, Jette N. The incidence and prevalence of Huntington's disease: a systematic review and meta-analysis. Mov Disord. 2012 Aug;27(9):1083-91.

9 Panas M, Karadima G, Vassos E, Kalfakis N, Kladi A, Christodoulou K, et al. Huntington's disease in Greece: the experience of 14 years. Clin Genet. 2011 Dec;80(6):586-90.

10 Kokmen E, Ozekmekçi FS, Beard CM, O'Brien PC, Kurland LT. Incidence and prevalence of Huntington's disease in Olmsted County, Minnesota (1950 through 1989). Arch Neurol. 1994 Jul;51(7):696-8.

11 Sackley C, Hoppitt TJ, Calvert M, Gill P, Eaton B, Yao G, et al. Huntington's disease: current epidemiology and pharmacological management in UK primary care. Neuroepidemiology. 2011;37(3-4):216-21.

12 Castilhos RM, Santos JAD, Augustin MC, Pedroso JL, Barsottini O, Saba R, et al. Minimal prevalence of Huntington's disease in the South of Brazil and instability of the expanded CAG tract during intergenerational transmissions. Genet Mol Biol. 2019 Apr-Jun;42(2): 329-36.

13 Wexler NS, Collett L, Wexler AR, Rawlins MD, Tabrizi SJ, Douglas I, et al. Incidence of adult Huntington's disease in the UK: a UKbased primary care study and a systematic review. BMJ Open. 2016 Feb 23;6(2):e009070.

14 Almqvist EW, Elterman DS, MacLeod PM, Hayden MR. High incidence rate and absent family histories in one quarter of patients newly diagnosed with Huntington disease in British Columbia. Clin Genet. 2001;60(3) 198-205.
15 Ohlmeier C, Saum K-U, Galetzka W, Beier D, Gothe H. Epidemiology and health care utilization of patients suffering from Huntington's disease in Germany: real world evidence based on German claims data. BMC Neurology. 2019;19(1):318.

16 Carrassi E, Pugliatti M, Govoni V, Sensi M, Casetta I, Granieri E. Epidemiological study of Huntington's Disease in the province of Ferrara, Italy. Neuroepidemiology. 2017; 49(1-2):18-23.

17 Ramos-Arroyo MA, Moreno S, Valiente A. Incidence and mutation rates of Huntington's disease in Spain: experience of 9 years of direct genetic testing. J Neurol Neurosurg Psychiatry. 2005 Mar;76(3):337-42.

18 Chen YY, Lai CH. Nationwide populationbased epidemiologic study of Huntington's disease in Taiwan. Neuroepidemiology. 2010; 35(4):250-4.

19 Squitieri F, Maffi S, Al Harasi S, Al Salmi Q, D'Alessio B, Capelli G, et al. Incidence and prevalence of Huntington disease (HD) in the Sultanate of Oman: the first Middle East postHTT service-based study. J Neurol Neurosurg Psychiatry. 2020 Dec;91(12):1359-60.

20 Bruzelius E, Scarpa J, Zhao Y, Basu S, Faghmous JH, Baum A. Huntington's disease in the United States: variation by demographic and socioeconomic factors. Mov Disord. 2019 Jun;34(6):858-65.

21 Exuzides A, Crowell V, Reddy SR, Chang E, Yohrling G. Epidemiology of Huntington's disease (HD) in the US Medicare population. Neurology. 2020;94(15 Suppl 1):670.

22 Rawlins M. Huntington's disease out of the closet? Lancet. 2010 Oct 23;376(9750):13723.

23 Evans SJ, Douglas I, Rawlins MD, Wexler NS, Tabrizi SJ, Smeeth L. Prevalence of adult Huntington's disease in the UK based on diagnoses recorded in general practice records. J Neurol Neurosurg Psychiatry. 2013 Oct; 84(10):1156-60.

24 Fisher ER, Hayden MR. Multisource ascertainment of Huntington disease in Canada: prevalence and population at risk. Mov Disord. 2014 Jan;29(1):105-14.

25 Squitieri F, Griguoli A, Capelli G, Porcellini A, D'Alessio B. Epidemiology of Huntington disease: first post-HTT gene analysis of prevalence in Italy. Clin Genet. 2016 Mar;89(3): 367-70.

26 Baig SS, Strong M, Quarrell OW. The global prevalence of Huntington's disease: a systematic review and discussion. Neurodegener Dis Manag. 2016;6(4):331-43.

27 Wexler A. Stigma, history, and Huntington's disease. Lancet. 2010 Jul 3;376(9734):18-9.

28 Kruijshaar ME, Barendregt JJ, van de PollFranse LV. Estimating the prevalence of breast cancer using a disease model: data problems and trends. Popul Health Metr. 2003;1(1):5.
29 Barendregt JJ, Baan CA, Bonneux L. An indirect estimate of the incidence of non-insulindependent diabetes mellitus. Epidemiology. 2000;11(3):274-9.

30 Pignon B, Schürhoff F, Baudin G, Tortelli A, Ferchiou A, Saba G, et al. Relationship between incidence and prevalence in psychotic disorders: an incidence-prevalence-mortality model. Int J Methods Psychiatr Res. 2018; 27(4):e1719.

31 United Nations Population Division. World Population Prospects 2019. [Accessed 8 January] Available from: https://population.un. org/wpp/.

32 Wexler NS. Huntington's disease: advocacy driving science. Annu Rev Med. 2012;63(1): $1-22$.

33 Landwehrmeyer GB, Fitzer-Attas CJ, Giuliano JD, Gonçalves N, Anderson KE, Cardoso F, et al. Data analytics from Enroll-HD, a global clinical research platform for Huntington's disease. Mov Disord Clin Pract. 2017 Mar-Apr;4(2):212-24.

34 Honrath P, Dogan I, Wudarczyk O, Görlich KS, Votinov M, Werner CJ, et al. Risk factors of suicidal ideation in Huntington's disease: literature review and data from Enroll-HD. J Neurol. 2018 Nov;265(11):2548-61.

35 Jacobs M, Hart EP, van Zwet EW, Bentivoglio AR, Burgunder JM, Craufurd D, et al. Progression of motor subtypes in Huntington's disease: a 6-year follow-up study. J Neurol. 2016 Oct;263(10):2080-5.

36 Paulsen JS, Nehl C, Hoth KF, Kanz JE, Benjamin $\mathrm{M}$, Conybeare R, et al. Depression and stages of Huntington's disease. J Neuropsychiatry Clin Neurosci. 2005;17(4):496-502.

37 Rawlins MD, Wexler NS, Wexler AR, Tabrizi SJ, Douglas I, Evans SJ, et al. The prevalence of Huntington's disease. Neuroepidemiology. 2016;46(2):144-53.

38 Yohrling G, Raimundo K, Crowell V, Lovecky D, Vetter L, Seeberger L. Prevalence of Huntington's disease in the US. Neurology. 2020; 94(15 Suppl 1):954.

39 Uhlmann WR. The woman who walked into the sea: Huntington's and the making of a genetic disease. Am J Hum Genet. 2010;86(6): 830-1.

40 Vicente E, García-Amigot F, Gaston M, Nuin-Villanueva M, Hernández B, Ardanaz E, et al. J3W Prevalence of Huntington disease in Navarra (Spain). Sensitivity and positive predictive value of different sources of asecertaiment [sic]. J Neurol Neurosurg Psychiatry. 2014;85:A77.

41 Morrison PJ, Harding-Lester S, Bradley A. Uptake of Huntington disease predictive testing in a complete population. Clin Genet. 2011 Sep;80(3):281-6. 